\title{
Análise Fatorial Confirmatória da Versão Reduzida da Escala de Impulsividade Barratt para Adolescentes
}

\author{
Alice Rodrigues Willhelm (D), Anderson Siqueira Pereira (D), Rosa Maria Martins de Almeida ${ }^{1}$ (D) \\ Universidade Federal do Rio Grande do Sul, Porto Alegre-RS, Brasil
}

\section{RESUMO}

O objetivo geral foi avaliar a adequação da estrutura fatorial da versão brasileira da Barratt (BIS-11) para adolescentes de ambos os sexos e sugerir versão abreviada da escala com melhores propriedades psicométricas. Foram avaliados 304 adolescentes com idades entre 13 e 18 anos da cidade de Porto Alegre. Foi usada Escala de Impulsividade Barratt. Foi possível observar baixo índice de ajuste do modelo brasileiro em comparação com os demais e bom índice do modelo português. Porém, foi proposto uma versão reduzida da escala, totalizando 12 itens. Esta versão apresentou índices de ajuste adequados. O alfa de Cronbach corrigido obteve valor de 0,974. Este estudo apresenta boa análise fatorial que identifica três fatores da escala original para a população de adolescentes de ambos os sexos em versão abreviada da escala.

Palavras-chave: impulsividade; adolescência; avaliação; escala de impulsividade Barratt.

ABSTRACT - Confirmatory factor analysis of the reduced version of the Barratt Impulsivity Scale for adolescents The general aim was to evaluate the adequacy of the factorial structure of the Brazilian version of the Barratt Impulsivity Scale (BIS-11) for adolescents of both sexes and to suggest an abridged version of the scale with better psychometric properties. A total of 304 adolescents aged 13 to 18 years of the city of Porto Alegre were evaluated. The Barratt Impulsivity Scale was used. It was possible to observe a low fit index for the Brazilian model compared to the other indices and a good index for the Portuguese model. However, a reduced version of the scale was proposed, totaling 12 items. This version had adequate fit indices. The corrected Cronbach's alpha was 0.974 . This study presents a good factorial analysis that identifies three factors of the original scale for the population of adolescents of both sexes in an abbreviated version of the scale.

Keywords: impulsiveness; adolescence; evaluation; Barratt impulsiveness scale.

\section{RESUMEN - Análisis factorial confirmatorio de la versión reducida de la escala de impulsividad Barratt para adolescentes \\ El objetivo de la investigación fue evaluar la adecuación de la estructura factorial de la versión brasileña de Barratt (BIS-11) para adolescentes de ambos sexos y sugerir una versión abreviada de la escala con mejores propiedades psicométricas. Fueron evaluados 304 adolescentes con edades entre 13 y 18 años de la ciudad de Porto Alegre. Se utilizó la Escala de Impulsividad Barratt. Se observó que la tasa de ajuste bajo del modelo brasileño en comparación con los demás y buen índice del modelo portugués. Sin embargo, se propuso una versión reducida de la escala, totalizando en 12 ítems. Esta versión presentó índices de ajuste adecuados. El alfa de Cronbach corregido obtuvo un valor de 0,974. Este estudio presenta un buen análisis factorial que identifica tres factores de la escala original para la población de adolescentes de ambos sexos en la versión abreviada de la escala. \\ Palabras clave: impulsividad; adolescencia; evaluación; escala de impulsividad Barratt.}

Os comportamentos impulsivos são considerados um fenótipo bastante abrangente e multifacetado, que pode englobar vários tipos de comportamentos e expressões cognitivas, por isso, é um fator complexo (Moeller, Barratt, Dougherty, Schmitz, \& Swann, 2001). Apesar de ser considerado um termo heterogêneo, a impulsividade pode ser definida como comportamentos rápidos, impensados, que são feitos sem a avaliação do contexto e que contemplam a incapacidade de inibir uma resposta e adiar gratificações (Moeller et al., 2001; Patton, Stanford, \& Barrat, 1995; Swann, Bjork, Moeller, \& Dougherty, 2002).

Quando se relaciona impulsividade com a fase da infância ou da adolescência, pensa-se tanto em problemas externalizantes, tais como comportamentos agressivos (de Almeida et al., 2014; Mathias, Marsh-Richard, \& Dougherty, 2008), hiperatividade (Nilsson et al., 2016), uso ou abuso de álcool e drogas (Crews \& Boettiger, 2009; 
de Almeida et al., 2014; Ryan, MacKillop, \& Carpenter, 2013), como problemas internalizantes, por exemplo, dificuldades de aprendizado, depressão e ansiedade (Cataldo, Nobile, Lorusso, Battaglia, \& Molteni, 2005). Porém, apesar de se observar a relação da impulsividade, diversas questões comportamentais importantes, ainda existe a escassez de instrumentos de autorrelato que avaliem esse fenótipo em crianças e adolescentes (Chahin, Cosi, Lorenzo-Seva, \& Vigil-Colet, 2010; Diemen, Szobot, Kessler, \& Pechansky, 2007).

A Barratt (BIS-11) é um instrumento de autorrelato amplamente utilizado na literatura para a avaliação da impulsividade e hoje possui normatização para crianças e adolescentes com dados de diversos países (Bhat, Roopesh, Bhaskarapillai, \& Benegal, 2018; Chahin et al., 2010; Diemen et al., 2007; Martínez-Loredo, FernándezHermida, Fernández-Artamendi, Carballo, \& GarcíaRodríguez, 2015; Pechorro, Ayala-Nunes, Ray, Nunes, \& Gonçalves, 2016). Originalmente, a escala Barratt foi desenvolvida para avaliar impulsividade como traço de personalidade unidimensional, porém, a versão 11 apresentou três fatores: motora, cognitiva ou atencional e não planejamento (Patton et al., 1995). O fator 1, impulsividade atencional, foi definido como falta de foco na tarefa em andamento e composto por dois fatores de primeira ordem, atenção e instabilidade cognitiva; o fator 2, impulsividade motora, foi definida como ação sem inibição de respostas prepotentes ou contínuas e inclui dois fatores, perseverança e representação; o fator 3 , impulsividade por não planejamento, foi definida como orientação para o presente, e não para o futuro, e incluiu autocontrole e complexidade cognitiva (Patton et al., 1995).

A versão americana da BIS-11 foi traduzida para pelo menos 11 idiomas, a escala está disponível e chinês (Yao et al., 2007), francês (Bayle et al., 2000), alemão (Hartmann, Rief, \&

Hilbert, 2011), italiano (Fossati, Di Ceglie, Acquarini, \& Barratt, 2001), japonês (Someya et al., 2001), coreano (Lee \& Kweon, 2014), português (Malloy-Diniz et al., 2010), turco (Güleç et al., 2008), entre outros. Porém, existe uma gama de estudos que não conseguiu replicar a estrutura fatorial de três fatores desenvolvida por Patton et al. (1995) com amostra adolescente (Diemen et al., 2007; Fossati, Barratt, Acquarini, \& Di Ceglie, 2002; Hartmann, Rief, \& Hilbert, 2011).

Tal questão acontece devido à dificuldade de replicar uma versão com os três fatores propostos pelo estudo original (Diemen et al., 2007). Apesar de encontrarem bons índices de consistência interna, as análises exploratórias da BIS-11 para adolescentes identificam os fatores, porém, diferentes daqueles da escala original em inglês (Diemen et al., 2007; Hartmann et al., 2011). Os três fatores propostos pela escala original (impulsividade atencional, motora e por não planejamento) são observados nas escalas traduzidas, porém pode existir um viés cultural relacionado, visto que, em cada país, as análises exploratórias identificaram itens diferentes dentro de cada fator.

No Brasil, ainda há poucos estudos sobre a adaptação ou normatização da escala, sendo esses estudos apenas com a população masculina dessa fase da vida (Diemen et al., 2007; Vieira, 2014). E ainda ressalta-se que o estudo que realizou a adaptação cultural da escala apresenta nível de consistência interna satisfatória $(0,62)$, a solução obteve resultado diferente da versão original, e os autores afirmaram a não validação dos três fatores, logo recomendou-se o uso da escala apenas para escore total (Diemen et al., 2007).

Nesse sentido, autores apontaram a necessidade de produzir instrumentos de avaliação mais eficientes e mais abreviados (Coutlee, Politzer, Hoyle, \& Huettel, 2014; Steinberg, Sharp, Stanford, \& Tharp, 2013). O estudo de Coutlee et al. (2014) apresentou uma escala reduzida, chamada de ABbreviated Impulsiveness Scale (ABIS), que é composta por 13 itens e, apesar de simplificada, mantém a estrutura dos três fatores proposta por Patton et al., (1995).

Além disso, percebeu-se a necessidade de um estudo brasileiro que apresentasse métodos analíticos robustos e apropriados. Por isso, o presente estudo optou por utilizar o estimador Weighted Least Squares Means and Variance Adjusted (WLSMV), que é um dos mais apropriados para dados de tipo categórico ordinal, o que pode representar uma vantagem em relação a estudos anteriores. Em contraste, o estudo de Patton et al. (1995) utilizou métodos menos refinados. De fato, estudos revelam que não usar um estimador apropriado pode produzir soluções fatoriais com um número incorreto de fatores e com estimativas paramétricas enviesadas (Kam \& Meyer, 2015; Weydmann, Hauck Filho, \& Bizarro, 2020).

Refletindo também sobre a amostra, estudos que avaliaram adolescentes brasileiros, ocuparam-se da população do sexo masculino, não apresentando dados de validade para amostras de adolescentes do sexo feminino (Diemen et al., 2007; Vieira, 2014). Enquanto estudos internacionais já realizam pesquisas que apresentam resultados para ambos os sexos (Fossati et al., 2002; Hartmann et al., 2011; Pechorro, Oliveira, Gonçalves, \& Jesus, 2018). Nesse sentido, o objetivo geral deste estudo foi sugerir uma versão abreviada da escala com melhores propriedades psicométricas com o uso de um estimador apropriado (WLSMV) para adolescentes brasileiros de ambos os sexos. Também foi proposto utilizar as estruturas fatoriais da versão brasileira e da versão portuguesa reduzida da BIS-11 (Pechorro et al., 2018) com a amostra deste estudo para verificar a adequação delas e, com base nisso, sugerir nova estrutura de fatores.

\section{Método}

\section{Amostra}

Foram avaliados 304 adolescentes de ambos os sexos com idades entre 13 e 18 anos de escolas públicas e 
privadas da cidade de Porto Alegre. Os critérios de inclusão foram ter entre 13 e 18 anos e estar devidamente matriculado e estudando. O critério de exclusão para o estudo foi apresentar problemas visuais (não corrigidos) e/ou cognitivos que impedissem a avaliação. Dados de descrição da amostra, como sexo e média de idade, podem ser observados na Tabela 1.

Tabela 1

Dados de Descrição da Amostra

\begin{tabular}{lc}
\hline Sexo & \\
$\quad$ Masculino & $n=188$ \\
Feminino & $n=116$ \\
Idade & $13-18$ \\
Média (DP) & $15,36(1,16)$ \\
\hline
\end{tabular}

Nota. $n=$ número de participantes; $D P=$ desvio padrão

\section{Procedimentos de Coletas e Éticos}

$\mathrm{O}$ estudo respeitou as normas estabelecidas para a realização de pesquisas com seres humanos pelo Conselho Federal de Psicologia - Resolução no. 010/12 e pelo Conselho Regional de Saúde (2012) - Resolução n ${ }^{\circ}$. 466/2012 tendo sido submetida e aprovada pelo Comitê de Ética da UFRGS. Os responsáveis assinaram no Termo de Consentimento Livre e Esclarecido (TCLE) antes da avaliação, e os participantes assinaram o Termo de Assentimento Livre e Esclarecido, concordando em participar da pesquisa (TALE).

A busca por participantes ocorreu por meio de sete escolas de Porto Alegre. Todos os alunos desses colégios que estavam dentro da faixa etária estudada foram convidados a participar da pesquisa. Os interessados levaram o TCLE para casa para que pudessem coletar a assinatura e autorização dos responsáveis, assim que o termo retornou à escola assinado, o aluno foi chamado para participar. Os adolescentes foram avaliados na escola durante o turno de aulas em um momento cedido pela direção e professores, de forma que não prejudicasse tarefas acadêmicas importantes, em uma sala reservada, sem ruídos e com boa iluminação. A avaliação foi feita de forma individual porque contemplou outros instrumentos que fazem parte de um estudo maior.

\section{Instrumento}

Barratt Impulsiveness Scale (BIS). A BIS é um questionário que pretende avaliar impulsividade. É o instrumento mais utilizado para medir impulsividade desde sua primeira versão em 1959 (Stanford et al., 2009). Contém 30 questões tipo Likert, sendo as opções de resposta (1) nunca/raramente; (2) às vezes; (3) frequentemente; (4) quase sempre/sempre. A pontuação pode ir de 30 a 120 e não existe um ponto de corte. Como mencionado anteriormente, as questões medem três fatores de impulsividade: motora, não planejamento e desatenção. A BIS-youth, versão em inglês adaptada para adolescentes, já foi utilizada em outros estudos e 13 itens da versão adulta foram revistos para tornar a escala adequada para adolescentes (ex.: "Eu planejo um trabalho seguro" foi trocado por "Eu planejo meus estudos antes do tempo") e outros 17 itens permaneceram idênticos à versão original adulta (Niv, Tuvblad, Raine, Wang \& Baker, 2012). A versão utilizada neste estudo é a de Diemen et al. (2007) e foi adaptada de Malloy-Diniz et al. (2010).

\section{Análise de Dados}

Para a análise das propriedades psicométricas, realizou-se análises fatoriais confirmatórias (AFC) da estrutura fatorial portuguesa da BIS-11 por meio do software Mplus, utilizando o método de Weighted Least Squares Means and Variance Adjusted (WLSMV). Foram considerados valores adequados de ajuste Comparative Fit Index (CFI) e Tucker-Lewis Index (TLI) acima de 0,9 e Root Mean Square Error of Approximation (RMSEA) com valores abaixo de 0,08 como um ajuste aceitável e valores abaixo de 0,05 como bom ajustamento, todos utilizando $90 \%$ de intervalo de confiança (Hu \& Bentler, 1999). Para análises da consistência interna das escalas, foi calculado o alfa de Cronbach, sendo considerados valores entre 0,7 e 0,9 como ideais. Nos casos em que as escalas possuíam menos de sete itens, optou-se pela utilização da fórmula Spearman-Brown, pois o alfa tende a ser influenciado negativamente em escalas com poucos itens (Swailes \& McIntyre-Bhatty, 2002), e a fórmula Spearman-Brown permite predizer o alfa com o dobro de itens, corrigindo esse problema.

\section{Resultados}

Foram testados os índices de ajustamento do modelo de Pechorro et al. (2018) e Diemen et al. (2007) e, como a estrutura fatorial da versão portuguesa da BIS-11 e da brasileira não apresentaram bons índices de ajuste, decidiu-se por realizar uma nova análise do conteúdo dos itens propostos por Diemen et. al. (2007). Os itens com baixos índices de ajuste foram excluídos, então 11 itens foram excluídos $(04,05,11,13,14,18,20,23,24,25,28)$.

Com os 19 itens restantes, foi realizada uma AFC utilizando os três fatores originais da escala (Atenção, Motora e Planejamento), porém, essa versão não apresentou bons índices de ajustamento. Assim, foram analisados os resultados desta análise e foram escolhidos os quatro itens com melhores cargas fatoriais em cada fator para a construção de uma versão reduzida da escala, totalizando em 12 itens. Essa versão apresentou índices de ajuste adequados. Além disso, foram consultados dois juízes independentes para avaliar os itens teoricamente, no sentido de cada item poder contemplar, de formas diferentes, o fator no qual está inserido. Os índices de ajuste de todas as versões podem ser observados na Tabela 2 
e as cargas fatoriais e consistências internas da versão reduzida podem ser observados na Tabela 3 . O alfa de
Cronbach total da escala ficou no valor de 0,759 e o alfa corrigido obteve o valor de 0,974 .

Tabela 2

Índices de Ajuste dos Modelos Testados da BIS-11

\begin{tabular}{lcccc}
\hline \multicolumn{1}{c}{ Modelo } & $\begin{array}{c}\chi^{2} \text { (graus de } \\
\text { liberdade) }\end{array}$ & CFI & TLI & RMSEA (90\% CI) \\
\hline $\begin{array}{l}\text { Modelo original BIS-11 } \\
\text { (30 itens em 3 fatores) }\end{array}$ & $1861,247(435)$ & 0,670 & 0,640 & $0,063(0,057-0,068)$ \\
$\begin{array}{l}\text { Modelo Português reduzido } \\
\text { (18 itens em 3 fatores, porém sem item 3, 20 e 05) }\end{array}$ & $947,664(105)$ & 0,893 & 0,871 & $0,059(0,046-0,071)$ \\
Versão com 19 itens & $1526,268(171)$ & 0,883 & 0,865 & $0,059(0,050-0,069)$ \\
Versão com 12 itens & $1003,374(66)$ & 0,950 & 0,935 & $0,055(0,039-0,072)$ \\
\hline
\end{tabular}

Notas. BIS-11=Escala de Barratt; CFI=Comparative Fit Index; TLI=Tucker-Lewis Index; RMSEA=Root Mean Square Error of Approximation; $\mathrm{CI}=$ consistência interna

Tabela 3

Cargas Fatoriais dos Itens da Versão Reduzida da Barratt

\begin{tabular}{|c|c|c|c|c|c|}
\hline Itens & F1 & F2 & F3 & Alfa & $\begin{array}{c}\text { Alfa } \\
\text { corrigido }\end{array}$ \\
\hline Atenção & & & & 0,558 & 0,835 \\
\hline 7. Eu me concentro com facilidade ${ }^{(R)}$ & 0,654 & & & & \\
\hline 10. Eu costumo pensar com cuidado em tudo $(\mathrm{R})$ & 0,566 & & & & \\
\hline 16. Eu me canso com facilidade tentando resolver problemas mentalmente, de cabeça. & 0,442 & & & & \\
\hline 29. Eu me sinto inquieto em aulas e palestras. & 0,400 & & & & \\
\hline Motor & & & & 0,691 & 0,899 \\
\hline 2. Eu faço coisas sem pensar. & & 0,647 & & & \\
\hline 6. Eu sou uma pessoa controlada ${ }^{(\mathrm{R})}$ & & 0,597 & & & \\
\hline 12. Eu falo as coisas sem pensar. & & 0,688 & & & \\
\hline 15. Eu faço as coisas no impulso. & & 0,656 & & & \\
\hline Planejamento & & & & 0,522 & 0,814 \\
\hline 1. Eu planejo tarefas com cuidado ${ }^{(\mathrm{R})}$ & & & 0,653 & & \\
\hline 8. Eu tenho facilidade para economizar dinheiro ${ }^{(\mathrm{R})}$ & & & 0,344 & & \\
\hline 17. Eu me cuido para não ficar doente ${ }^{(\mathrm{R})}$ & & & 0,505 & & \\
\hline 22. Eu termino o que começo ${ }^{(\mathrm{R})}$ & & & 0,547 & & \\
\hline
\end{tabular}

Notas. ${ }^{(R)}$ equivale a itens que são calculados de forma inversa, quando maior a pontuação, menor o nível de impulsividade. Abreviações. F1=Cargas fatoriais no Fator 1; F2=Cargas fatoriais no Fator 2; F3=Cargas fatoriais no Fator 3

\section{Discussão}

Um dos objetivos deste estudo foi utilizar as estruturas fatoriais da versão brasileira e da versão portuguesa reduzida da BIS-11 (Pechorro et al., 2018) com a amostra desse estudo para verificar a adequação delas e, nesse sentido, os resultados demonstraram que essas versões não possuem bons índices de ajustamento para a amostra deste estudo. Os resultados com população adolescente apresentam dificuldade de encontrar nível de consistência interna, como, por exemplo, o estudo que se propôs a fazer a adaptação da escala original para adolescentes brasileiros do sexo masculino, que não encontrou bons índices para as subescalas da BIS-11 (Diemen et. al., 2007), bem como também não obteve bons resultados com a amostra do presente estudo. Tal questão pode se dar por fatores culturais, visto que em países diferentes, as análises exploratórias identificaram itens diferentes dentro de casa fator.

Observando ainda os resultados, foi possível perceber que a versão portuguesa reduzida apresenta melhores índices de ajustamento do que a versão brasileira 
adaptada para a população adolescente. Tal questão corrobora a ideia de Coutlee et al. (2014) e Steinberg, Sharp, Stanford e Tharp (2013) de produzir instrumentos que possam avaliar um construto de forma abreviada e mais eficaz, visto que uma estrutura semelhante com duas amostras distintas (brasileira e portuguesa) produziu resultados mais promissores.

Em decorrência disso, este estudo também objetivou descobrir alternativas para encontrar melhores propriedades psicométricas da BIS-11 e, observando uma melhora dos índices na versão abreviada portuguesa, foi proposto uma nova versão que pudesse avaliar os três fatores propostos por Patton et al. (1995). Foi sugerida uma versão de 12 itens que foram distribuídos igualmente entre os fatores: motor, atenção e planejamento. A adaptação manteve itens da versão brasileira adaptada para adolescentes e demonstrou que os itens selecionados obtiveram boas cargas fatoriais dentro de cada fator.

É importante salientar que alguns itens da versão adaptada obtiveram carga fatorial boa em um fator que não seu original, logo, propôs-se a troca do item para o subtipo de impulsividade. Por exemplo, o item "Eu costumo pensar com cuidado em tudo" foi classificado como impulsividade de planejamento na versão adaptada de Diemen et al. (2007) e, neste estudo, obteve boa carga fatorial dentro do subtipo de Impulsividade atencional. O mesmo ocorreu com o item "Eu me canso com facilidade tentando resolver problemas mentalmente, de cabeça", que também obteve melhor carga fatorial no subtipo Atenção e, ambos os itens foram trocados de subtipo de Impulsividade. Assim como os itens acima mencionados, os subitens 6 e 12 ("Eu sou uma pessoa controlada" e "Eu falo as coisas sem pensar" respectivamente) obtiveram peso no subtipo Planejamento na versão de Diemen et al. (2007), porém, neste estudo, os itens obtiverem melhores cargas em outro subtipo, na Impulsividade motora, logo, também foram alterados na versão reduzida.

Os resultados sugerem que a alteração da escala não se deu apenas na redução de itens da versão original de Diemen et al. (2007), mas também demonstrou a importância de a escala ser estudada novamente para a observação de alterações em sua estrutura. Alguns itens atualmente podem não ter mais tanto peso em um fator específico, ou seja, aquele item pode não estar mais avaliando o subtipo de impulsividade que se propôs a avaliar e essa alteração se torna necessária para que a escala possa seguir avaliando de forma fiel os três subtipos propostos por Patton et al. (1995).

Além disso, a amostra deste estudo avaliou adolescentes de ambos os sexos, ou seja, a escala reduzida também pode ser aplicada em meninas. Tal questão também foi um dos objetivos deste trabalho, que foi observado que versões que avaliam adolescentes brasileiros realizaram seu estudo com amostra masculina (Diemen et al., 2007; Vieira, 2014). Então, os resultados desde estudo propiciam a avaliação dessa população de ambos os sexos.
E ainda, ressalta-se que a população avaliada nesta pesquisa tinha idade entre 13 e 18 anos, captando propriamente a fase da adolescência, enquanto outros estudos propuseram a utilização da escala para a avaliação de sujeitos a partir dos 15 anos, abrangendo a utilização até os 20 anos de idade (Diemen et al., 2007; Vieira, 2014). A proposta do presente estudo foi avaliar adolescentes em idade escolar, em decorrência de esse ambiente ainda ser de grande importância na vida do sujeito, então toda a amostra foi avaliada dentro desse contexto. Logo, a versão reduzida da escala pode ser utilizada nessa faixa etária.

\section{Considerações Finais}

A Escala de Impulsividade Barratt é amplamente utilizada, já tendo sido traduzida para diversos idiomas, porém, ainda não há uma versão brasileira com boa análise fatorial que identifique os três fatores da escala original para a população de adolescentes de ambos os sexos. Por isso, este estudo apresenta resultados promissores nesse sentido, trazendo uma versão abreviada da escala que foi reorganizada e possui condições de avaliar meninos e meninas na fase da adolescência de forma geral, ainda mantendo uma estrutura de três fatores: impulsividade motora, por atenção e planejamento.

No entanto, é importante salientar algumas limitações do presente estudo, como um número de amostragem reduzido e a ausência de avaliação de outros grupos na fase da adolescência, como, por exemplo, grupos clínicos ou de outras regiões do Brasil. Por isso, torna-se importante a realização de mais estudos na área, que possam avaliar a estrutura proposta da versão reduzida com outras populações, bem como seria importante a utilização dessa versão reduzida com outros instrumentos que também avaliem impulsividade. Ainda assim, espera-se que este trabalho possa ter contribuído com a área e que possam ser o pioneiro no lançamento de uma versão reduzida da BIS-11 para adolescentes brasileiros.

\section{Agradecimentos}

Não há menções.

\section{Financiamento}

Todas as fontes de financiamento para elaboração e produção do estudo (coleta, análise e interpretação dos dados, bem como, escrita dos resultados no presente no manuscrito) foram fornecidas pelo projeto de pesquisa 'FAPERGS Pesquisador Gaúcho PqG processo 17/2551-0001149-0”".

\section{Contribuições dos autores}

Declaramos que todos os autores participaram da elaboração do manuscrito. Especificamente, o(s) autor(es) Alice Rodrigues Willhelm e Anderson Siqueira Pereira participou(ram) da redação inicial do estudo - conceitualização, investigação, visualização, o(s) autor(es) 
Alice Rodrigues Willhelm e Anderson Siqueira Pereira participou(ram) da análise dos dados, e o(s) autor(es) Rosa Maria Martins de Almeida participou(ram) da redação final do trabalho - revisão, edição e orientação.

\section{Disponibilidade dos dados e materiais}

Todos os dados e sintaxes gerados e analisados durante esta pesquisa serão tratados com total sigilo devido às exigências do Comitê de Ética em Pesquisa em Seres Humanos. Porém, o conjunto de dados e sintaxes que apoiam as conclusões deste artigo estão disponíveis mediante razoável solicitação ao autor principal do estudo.

\section{Conflito de interesses}

Os autores declaram que não há conflitos de interesses.

\section{Referências}

Bayle, F. J., Bourdel, M. C., Caci, H., Gorwood, P., Chignon, J. M., Ades, J., \& Loo, H. (2000). Factor analysis of french translation of the Barratt impulsivity scale (BIS-10). Canadian Journal of Psychiatry. Revue Canadienne de Psychiatrie, 45(2), 156-165. doi: $10.1177 / 070674370004500206$

Bhat, N. A., Roopesh, B. N., Bhaskarapillai, B., \& Benegal, V. (2018). Validation of the Barratt Impulsiveness Scale-short form among Indian adolescents. Asian Journal of Psychiatry, 37, 172-177. doi: 10.1016/j.ajp.2018.10.009

Cataldo, M. G., Nobile, M., Lorusso, M. L., Battaglia, M., \& Molteni, M. (2005). Impulsivity in depressed children and adolescents: a comparison between behavioral and neuropsychological data. Psychiatry Research, 136(2-3), 123-133. doi: 10.1016/j.psychres.2004.12.012

Chahin, N., Cosi, S., Lorenzo-Seva, U., \& Vigil-Colet, A. (2010). Stability of the factor structure of Barrat's Impulsivity Scales for children across cultures: a comparison of Spain and Colombia. Psicothema, 22(4), 983-989. Recuperado de http://www.psicothema.com/ $\mathrm{pdf} / 3830 . \mathrm{pdf}$

Coutlee, C. G., Politzer, C. S., Hoyle, R. H., \& Huettel, S. A. (2014). An Abbreviated Impulsiveness Scale constructed through confirmatory factor analysis of the Barratt Impulsiveness Scale Version 11. Archives of Scientific Psychology, 2(1), 1-12. doi: 10.1037/arc0000005

Crews, F. T., \& Boettiger, C. A. (2009). Impulsivity, frontal lobes and risk for addiction. Pharmacology, Biochemistry, and Behavior, 93(3), 237247. doi: 10.1016/j.pbb.2009.04.018

de Almeida, R. M. M., Trentini, L. B., Klein, L. A., Macuglia, G. R., Hammer, C., \& Tesmmer, M. (2014). Uso de álcool, drogas, níveis de impulsividade e agressividade em adolescentes do Rio Grande do Sul. Psico, 45(1), 65-72. doi: 0.15448/1980-8623.2014.1.12727

Diemen, L. von, Szobot, C. M., Kessler, F., \& Pechansky, F. (2007). Adaptation and construct validation of the Barratt Impulsiveness Scale (BIS 11) to Brazilian Portuguese for use in adolescents. Revista Brasileira de Psiquiatria, 29(2), 153-156. doi: 10.1590/S151644462006005000020

Fossati, A., Barratt, E. S., Acquarini, E., \& Di Ceglie, A. (2002). Psychometric properties of an adolescent version of the Barratt Impulsiveness Scale-11 for a sample of Italian high school students. Perceptual and Motor Skills, 95(2), 621-635. doi: 10.2466/pms.2002.95.2.621

Fossati, A., Di Ceglie, A., Acquarini, E., \& Barratt, E. S. (2001). Psychometric properties of an Italian version of the Barratt Impulsiveness Scale-11 (BIS-11) in nonclinical subjects. Journal of Clinical Psychology, 57(6), 815-828. doi: 10.1002/jclp.1051

Güleç, H., Tamam, L., Turhan, M., Karakuş, G., Zengin, M., \& Stanford, M. S. (2008). Psychometric Properties of the Turkish Version of the Barratt Impulsiveness Scale-11. Klinik Psikofarmakoloji Bulteni, 18(4), 251-258.

Hartmann, A. S., Rief, W., \& Hilbert, A. (2011). Psychometric Properties of the German Version of the Barratt Impulsiveness Scale, Version 11 (Bis-11) for Adolescents. Perceptual and Motor Skills, 112(2), 353-368. doi: 10.2466/08.09.10.PMS.112.2.353-368

Hu, L., \& Bentler, P. M. (1999). Cutoff criteria for fit indexes in covariance structure analysis: Conventional criteria versus new alternatives. Structural Equation Modeling: A Multidisciplinary Journal, 6(1), 1-55. doi: 10.1080/10705519909540118

Kam, C. C. S., \& Meyer, J. P. (2015). How careless responding and acquiescence response bias can influence construct dimensionality. Organizational research methods, 18(3), 512-541. doi:10.1177/1094428115571894

Lee, J. A., \& Kweon, Y. R. (2014). Factors affecting social competence in school-aged children according to alcohol consumption by parents. Journal of Korean Academy of Nursing, 44(5), 495-503. doi: 10.4040/jkan.2014.44.5.495

Malloy-Diniz, L. F., Mattos, P., Leite, W. B., Abreu, N., Coutinho, G., Paula, J. J. de, ... Fuentes, D. (2010). Translation and cultural adaptation of Barratt Impulsiveness Scale (BIS-11) for administration in Brazilian adults. Jornal Brasileiro de Psiquiatria, 59(2), 99-105. doi: 10.1590/S0047-20852010000200004

Martínez-Loredo, V., Fernández-Hermida, J. R., Fernández-Artamendi, S., Carballo, J. L., \& García-Rodríguez, O. (2015). Spanish adaptation and validation of the Barratt Impulsiveness Scale for early adolescents (BIS-11-A). International Journal of Clinical and Health Psychology, 15(3), 274-282. doi: 10.1016/j.ijchp.2015.07.002

Mathias, C. W., Marsh-Richard, D. M., \& Dougherty, D. M. (2008). Behavioral measures of impulsivity and the law. Behavioral Sciences E the Law, 26(6), 691-707. doi: 10.1002/bsl.841

Moeller, F. G., Barratt, E. S., Dougherty, D. M., Schmitz, J. M., \& Swann, A. C. (2001). Psychiatric aspects of impulsivity. American Journal of Psychiatry, 158(11), 1783-1793. doi: 10.1176/appi.ajp.158.11.1783

Nilsson, T., Falk, Ö., Billstedt, E., Kerekes, N., Anckarsäter, H., Wallinius, M., \& Hofvander, B. (2016). Aggressive Antisocial Behaviors Are Related to Character Maturity in Young Swedish Violent Offenders Independent of ADHD. Frontiers in Psychiatry, 7. doi: 10.3389/ fpsyt.2016.00185

Niv, S., Tuvblad, C., Raine, A., Wang, P., \& Baker, L. A. (2012). Heritability and longitudinal stability of impulsivity in adolescence. Behavior genetics, 42(3), 378-392. doi: 10.1007/s10519-011-9518-6

Patton, J. H., Stanford, M. S., \& Barratt, E. S. (1995). Factor structure of the Barratt impulsiveness scale. Journal of Clinical Psychology, 51(6), 768-774. doi: 10.1002/1097-4679(199511)51:6<768::AID-JCLP2270510607>3.0.CO;2-1 
Pechorro, P., Ayala-Nunes, L., Ray, J. V., Nunes, C., \& Gonçalves, R. A. (2016). The Barratt Impulsiveness Scale-11 Among a School Sample of Portuguese Male and Female Adolescents. Journal of Child and Family Studies, 25(9), 2753-2764. doi: 10.1007/s10826-016-0453-2

Pechorro, P., Oliveira, J. P., Gonçalves, R., \& Jesus, S. (2018). Propriedades psicométricas de uma versão reduzida da Escala de Impulsividade de Barratt-11 numa amostra escolar de adolescentes portugueses. Revista Iberoamericana de Diagnóstico y Evaluación Psicológica, 7, 157-170. doi: 10.21865/RIDEP47.2.11

Ryan, K. K., MacKillop, J., \& Carpenter, M. J. (2013). The relationship between impulsivity, risk-taking propensity and nicotine dependence among older adolescent smokers. Addictive Behaviors, 38(1), 1431-1434. doi: 10.1016/j.addbeh.2012.08.013

Stanford, M. S., Mathias, C. W., Dougherty, D. M., Lake, S. L., Anderson, N. E., \& Patton, J. H. (2009). Fifty years of the Barratt Impulsiveness Scale: An update and review. Personality and individual differences, 47(5), 385-395. doi: 10.1016/j.paid.2009.04.008

Someya, T., Sakado, K., Seki, T., Kojima, M., Reist, C., Tang, S. W., \& Takahashi, S. (2001). The Japanese version of the Barratt Impulsiveness Scale, 11th version (BIS-11): Its reliability and validity. Psychiatry and Clinical Neurosciences, 55(2), 111-114. doi: 10.1046/j.14401819.2001.00796.x

Steinberg, L., Sharp, C., Stanford, M. S., \& Tharp, A. T. (2013). New tricks for an old measure: The development of the Barratt Impulsiveness Scale-Brief (BIS-Brief). Psychological Assessment, 25(1), 216-226. doi: 10.1037/a0030550

Swailes, S., \& McIntyre-Bhatty, T. (2002). The "Belbin" team role inventory: reinterpreting reliability estimates. Journal of Managerial Psychology, 17(6), 529-536. doi: 10.1108/02683940210439432

Swann, A. C., Bjork, J. M., Moeller, F. G., \& Dougherty, D. M. (2002). Two models of impulsivity: relationship to personality traits and psychopathology. Biological Psychiatry, 51(12), 988-994. doi: 10.1016/S0006-3223(01)01357-9

Vieira, H. de M. (2014). Características psicométricas da escala de impulsividade de Barratt em adolescentes. (Dissertação de mestrado). Universidade Tuiuti do Paraná, Curitiba.

Weydmann, G., Hauck Filho, N., \& Bizarro, L. (2020). Acquiescent responding can distort the factor structure of the BIS/BAS scales. Personality and Individual Differences, 152, 109563. doi: 10.1016/j.paid.2019.109563

Yao, S., Yang, H., Zhu, X., Auerbach, R. P., Abela, J. R., Pulleyblank, R. W., \& Tong, X. (2007). An examination of the psychometric properties of the Chinese version of the Barratt Impulsiveness Scale, 11th version in a sample of Chinese adolescents. Perceptual and Motor Skills, 104(3), 1169-1182. doi: 10.2466/pms.104.4.1169-1182

\section{Sobre os autores}

Alice Rodrigues Willhelm é psicóloga (PUCRS), mestre em Psicologia pela Universidade Federal do Rio Grande do Sul (UFRGS) e, atualmente, é doutoranda na mesma universidade.

Anderson Siqueira Pereira é psicólogo (PUCRS), mestre em Psicologia pela Universidade Federal do Rio Grande do Sul (UFRGS) e, atualmente, é doutorando na mesma universidade.

Rosa Maria Martins de Almeida é psicóloga (UFRGS), mestre e doutora pela Universidade Federal do Rio Grande do Sul (UFRGS) com pós-doutorado na Tufts University (Boston, Estados Unidos). Atualmente é professora associada da Universidade Federal do Rio Grande do Sul (UFRGS).

\section{Como citar este artigo}

Willhelm et al. (2020). Análise Fatorial Confirmatória da Versão Reduzida da Escala de Impulsividade Barratt para Adolescentes. Avaliação Psicológica, 19(4), 461-467. http://dx.doi.org/10.15689/ap.2020.1904.17597.12 